\title{
Chess endgame news: understanding minor piece endgames
}

Article

Accepted Version

Study of Minor Piece Endgames

Haworth, G. (2019) Chess endgame news: understanding minor piece endgames. ICGA Journal, 41 (2). pp. 110-112. ISSN 1389-6911 doi: https://doi.org/10.3233/ICG-190106 Available at https://centaur.reading.ac.uk/82423/

It is advisable to refer to the publisher's version if you intend to cite from the work. See Guidance on citing.

Published version at: https://content.iospress.com/articles/icga-journal/icg190106

To link to this article DOI: http://dx.doi.org/10.3233/ICG-190106

Publisher: The International Computer Games Association

All outputs in CentAUR are protected by Intellectual Property Rights law, including copyright law. Copyright and IPR is retained by the creators or other copyright holders. Terms and conditions for use of this material are defined in the End User Agreement.

\section{www.reading.ac.uk/centaur}

\section{CentAUR}

Central Archive at the University of Reading

Reading's research outputs online 


\title{
Chess Endgame News: Understanding Minor Piece Endgames
}

\author{
Guy Haworth ${ }^{1}$ \\ Reading, UK
}

As Jacob Aagaard writes in his foreword to Understanding Minor Piece Endgames (Müller and Konoval, 2019), "every new book written by Karsten is an event for me". This book combines the insight and enthusiasm of the well-known and prolific endgame expert with the technical precision of Yakov Konoval's sub-8-man 'DTC' Depth to Conversion endgame tables. 'Man and machine' is an excellent combination and this is one of its finer feats of inexorable logic.

'UME' will be eagerly snapped up by professional players but it is also accessible to club players and enthusiasts such as this author. It is both informative and entertaining, certainly a worthy successor to Understanding Rook Endgames (Müller and Konoval, 2016). The board has no queens or rooks but sports at least one minor piece on the board: no side has more than one. The core domain ranges from KNKP to KXPPKX'P where $\mathrm{X}$ and $\mathrm{X}^{\prime}$ are $\mathrm{B}, \mathrm{N}$ or $\mathrm{P}$.

Demis Hassabis once opined (Sadler and Regan, 2019) that it is "the exquisite balance of the bishop and knight across the set of all positions, despite their vastly different mobility, that creates the dynamic tension in the game". Here, the book builds to Chapter 6 , some $40 \%$ of the whole, where finally, spiritual and temporal, bishop and knight, face off against each other. In preparation, we study the single knight against foot soldiers (ch. 1), the joust of knight against knight (ch. 2), the sole bishop (ch. 3) and the ecclesiastical struggle of bishop against bishop, whether in the same church on the same-colour squares (ch. 4) or not (ch. 5). The 'drawish ocb' situations, incidentally, seem to be enjoyed by ALPHAZERO.

The finer structure is clear once laid out but it takes a mathematical, rigorous and perceptive mind like Karsten's to realise what that structure should be. Beyond the focusing on specific endgames, the key characteristics and themes of each subdomain are defined and guide one's understanding. Pawns may be on specific files, may or may not be close, connected or 'passed', a minor piece may (not) have the advantage, bishops do (not) control the queening square etc. The contents list could perhaps have usefully gone one or two levels deeper than the chapter headings. The e-version of this review (Haworth, 2019) includes more detailed content listings and other summary data.

The many examples, over 500 of them, tend to focus on but are not limited to sub-8-man positions. Here, you will find the skill and artistry of many World Champions on display and sometimes they face each other: only Tigran Petrosian is missing from the line of succession. Fig. 1 highlights a few of the famous and/or complex gems. Some 340 'game' exercises and 46 pages of solutions also help to check understanding of the various points made. Fig. 2 provides a small selection for the reader.

The corpus of didactic positions and chess studies also provides material, see Figs. 3 and 4. Chapter 8 merely picks up on a few studies which have been cooked or corrected en passant. Chapter 7 touches on some opportunities missed by chess engines, 'limited' or at least challenged as they currently are by

\footnotetext{
${ }^{1}$ Communicating author: g.haworth@ reading.ac.uk
} 
'the curse of the knight' zugzwang positions, their search-horizons and by 'only' having 6-man EGTs. The now prominent CHIRON (\#8), FIRE (\#23), HOUdINI, (\#5/26), RYBKA (\#21), KOMODO (\#6/7/12), SHREDDER (\#10/11) and STOCKFISH (\#16/18) all have their moments in the shade but they are older, wiser and better informed now.
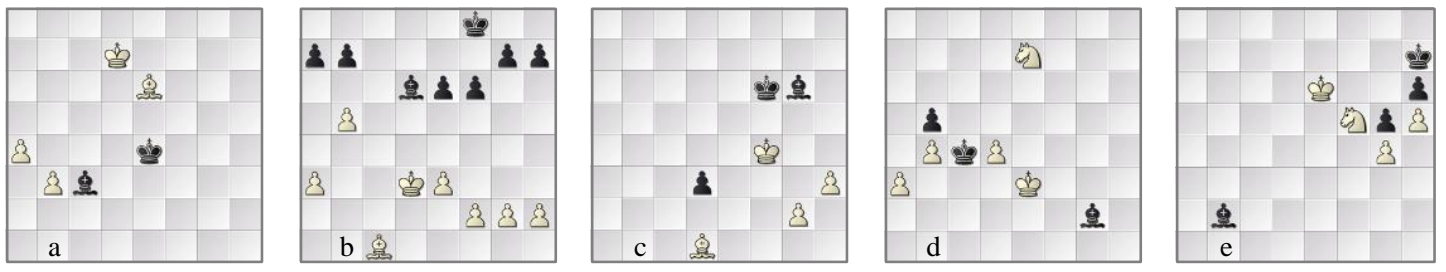

Fig. 1. (a) \#5.05 Shirov-Kramnik (1998) 50w; (b) \#3.77 Spassky-Fischer (WCC, 1972) 29b;

(c) \#4.10 Carlsen-Kramnik (2013) 46b; (d) \#6.191 Karpov-Kasparov (WCC, 1984) 66b; (e) \#6.192A Arkell-Burke, $70 b$.
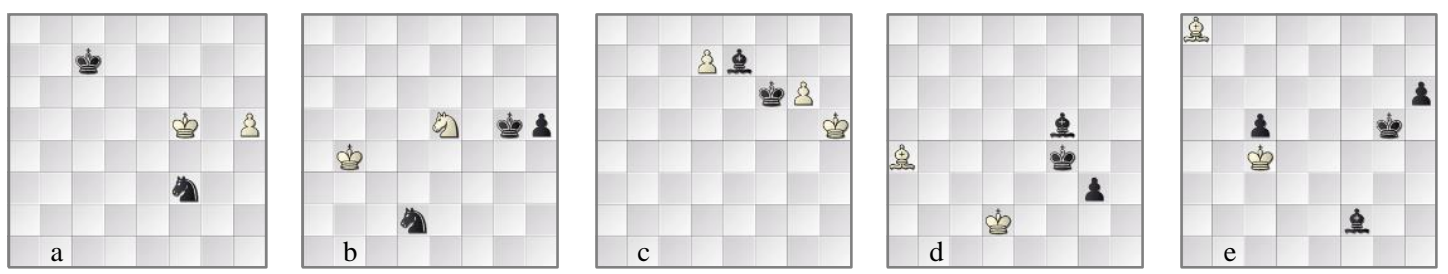

Fig. 2. Five exercises: (a) \#E1.01 btm and draw; (b) \#E2.01 btm and win; (c) \#E3.01 wtm and win; (d) \#E4.01 wtm and draw; (e) E5.01 wtm and draw.
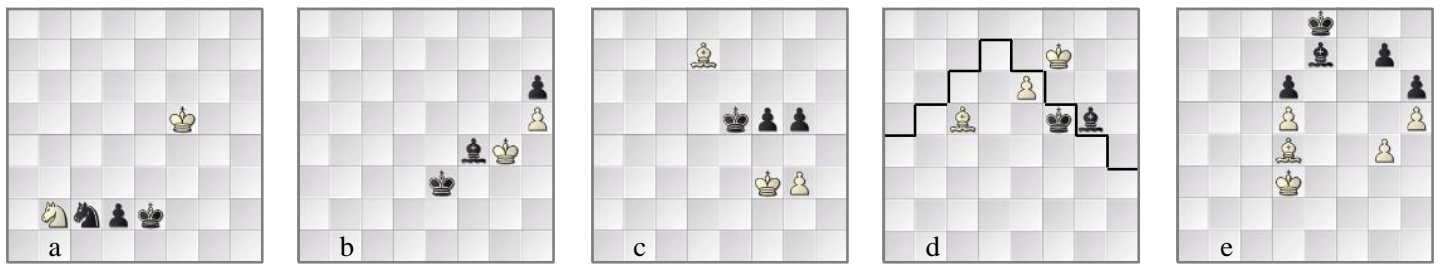

Fig. 3. (a) \#2.02 on Fine's Rule, (b) \#3.35 on Rauser's drawing zone, (c) \#3.47 on Averbakh's Barrier,

(d) \#4.03 on Centurini's Rule of 1856 and (e) \#4.38 on Capablanca's Rule.
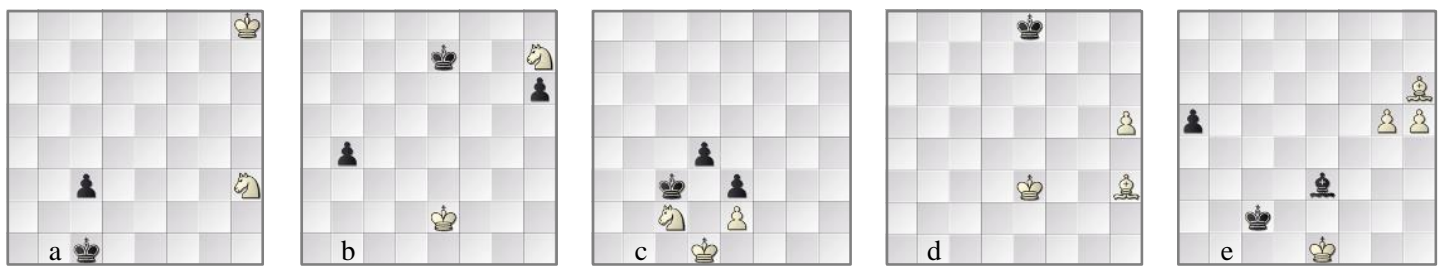

Fig. 4. Five studies: (a) \#1.04 Chéron (1924) wtm, =; (b) \#1.12 Grigoriev (1934) wtm, =;

(c) \#1.58 Réti (1924) wtm, 1-0; (d) \#E3.24 Troitzky (1896) wtm, 1-0; (e) \#4.36B Nunn (2013) wtm, 1-0.

Nearly forty 'longest-DTC wins' as in Fig. 5, show the full complexity of the various endgames, many of the lines being usefully commentated on. Endgame aficionados will also enjoy looking at the lines in the context of EGTs to metrics other than Konoval's 'DTC' Depth to Conversion. The Lomonosov (2012) DTM(ate) EGTs are now joined by the equally extensive and popular DTZ 50 " EGTs (de Man et al, 2018). Deep lines to different metrics can eventually diverge as they are pulled in different directions by their specific objectives. For example, do the three frustrated wins (\#2.47A KNPPknp, \#3.66 
KBPkppp and \#6.178 KNPPknp) let go of their 50-move-rule defence because of the given DTC-centric lines? ${ }^{2}$ Do the DTM- and DTC-minimaxing lines diverge and if so, where? What can be learned at the branch-point?
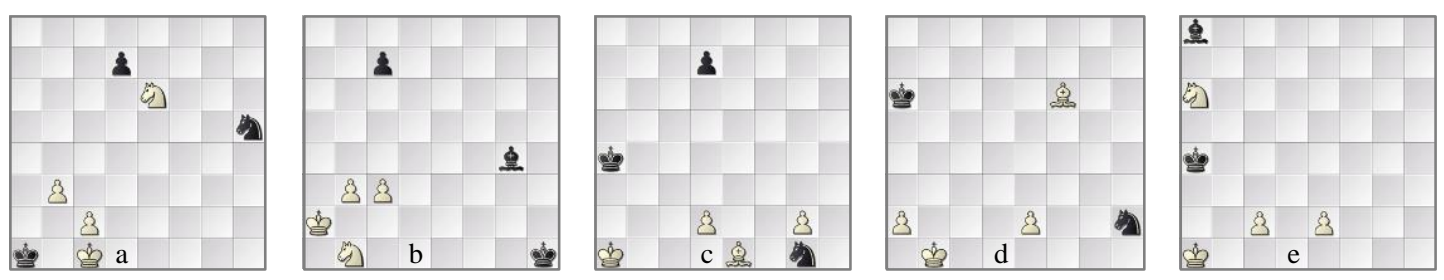

Fig. 5. The maxDTC-deepest 'UME' endgames, all 1-0: (a) \#2.47A wtm, dtc/m/z $=219 / 253 / 3$ ply;

(b) \#6.178 wtm, dtc/m/z = 203/263/4p; (c) \#6.118 wtm, dtc/m/z = 171/205/5p;

(d) \#6.22 btm, dtc/m/z = 162/182/16p; (e) \#6.35 btm, dtc/m/z = 156/176/10p.

Some broad hints are provided in the footnotes to the exercises ${ }^{34567}$ and studies ${ }^{89,10,11,12}$ above. Müller and Konoval have produced a definitive work here in UME, mastering a large subject with authority and organising it so that it is more accessible to the greater chess community. The result is attractive, fascinating, instructive, enriching - entirely as one would expect from this team. It is a pleasure to see items of beautiful glassware being skilfully drawn from the silica of endgame data.

\section{REFERENCES}

de Man, R., Fiekas, N. and Guo, B. (2018). https://tinyurl.com/icga007. Fiekas' interface to 'Syzygy formatted' de Man sub-7-man and Guo 7-man DTZ ${ }_{50}$ " EGTs.

Haworth, G. $M^{\mathrm{c}} \mathrm{C}$. (2019). http://centaur.reading.ac.uk/82423/. This item plus supplementary data on content, longest wins etc.

Lomonosov (2012). http://tb7.chessok.com/. Query interface to sub-8-man DTM EGTs.

Müller, K. and Konoval, Y. (2016). Understanding Rook Endgames. Gambit.

Müller, K. and Konoval, Y. (2019). Understanding Minor Piece Endgames. Russell Enterprises.

Sadler, M. and Regan, N. (2019). Game Changer. New in Chess.

\footnotetext{
2 \#2.47A, line 32b-82b 50mr-draws; \#3.66, 49. ... Ka3? (Ka5 needed); \#6.178, 9b-102w more than 50mr-draws.

${ }^{3}$ \#E1.01: not 74. ... Nh4+? 75. Kg5 Nf3+' 76. Kf6"'" Nh2; 74. Kd6" 75. h6 Ne5"'" 76. h7 Nf7"'" 77. Kf6 Nh8 ...

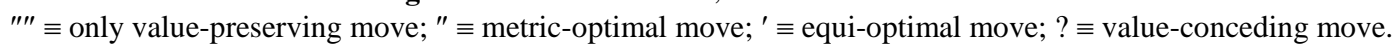
${ }^{4}$ \#E2.01: not 99. ... Kf5? 100. Kc3"'" Ne4+ 101. Kd4"'"; 99. ... Kf4"'" 100. Kc3 (Ng6+?) Nf3"'" 101. Ng6+ Kg5"'" ... 5 \#E3.01: not 78. Kh6? Bf8+"'" 79. Kh7 Ke7"'" 80. g7 Bxg7"'" 81. Kxg7; 78. d8=(Q/R/B)"'" Bxd8 79. Kh6"'" ...

6 \#E4.01: not 69. Ke2? Bd3+"'" 70. Ke1 Ke3" 71. Bc6" Be4"'" 72. Bxe4; 69. Ke1"'" Bd3 70. Bc6"'" Ke3 (Be4?) ...

7 \#E5.01: not 46. Bf3? h5"'" 47. Kd3 h4"'" 48. Ke2 Bd4"'"; 46. Kd3"'" Kf4 47. Ke2""' Bd4 48. Bf3"'" c4 49. Bh5 Kg5 ...

${ }^{8}$ \#1.04: 1. Nf4"'" (1. Ng1? Kd1"'" 2. Nh3 c2"; 1. Ng5? c2""' 2. Ne4 Kb2"'") 1. ... c2 2. Ne2+"'" Kd1 3. Nc3+"'" Kd2 Na2"'"

${ }^{9}$ \#1.12: 1. Kd3"'" Kf7 2. Kc4"'" Kg6 3. Nf8+"'" (3. Kxb4? h5"'") Kf5 4. Nd7"'" h5 5. Nc5"' h4 6. Nb3"'" h3 7. Nd2"'" ...

10 \#1.58: 1. Ne1"" Kb2 2. Nd3+" Kc3" 3. Nc1" Kb2" 4. Na2" Kb1" 5. Nb4" Kb2" 6. Nd5" Kb3" 7. Nc7" Kc3" ...

${ }^{11}$ \#E3.24: 1. Be6"'" Ke7" 2. h6"'" Kf6" 3. Bf5"' Kf7" 4. Bh7"' Kf6' 5. Kf4" Kf7 6. Kf5' Kf8' 7. Kf6" Ke8 8. Bg8 ...

12 \#4.36B: 1. Ke2"'" Bd2 2. Kf3"'" a4 3. Kg4"' a3 4. Bg7"'" Bc1 5. h6"'" Bb2 6. Kg3"'" (6. Kf4? zugzwang) 6. ... Kd3 7. Kf4"'" Kd2 8. Kf3"'" Kd3 ...
} 